\title{
Single fiber reflectance spectroscopy calibration
}

Xu U. Zhang

Anouk L. Post

Dirk J. Faber

Ton G. van Leeuwen

Henricus J. C. M. Sterenborg 


\section{Single fiber reflectance spectroscopy calibration}

\author{
Xu U. Zhang, ${ }^{a, *}$ Anouk L. Post, ${ }^{a}$ Dirk J. Faber, ${ }^{a}$ \\ Ton G. van Leeuwen, ${ }^{\mathrm{a}}$ and \\ Henricus J. C. M. Sterenborg ${ }^{a, b}$ \\ ${ }^{a}$ Academic Medical Center, University of Amsterdam, Department of \\ Biomedical Engineering and Physics, Amsterdam, The Netherlands \\ ${ }^{b}$ Netherlands Cancer Institute, Department of Surgery, Amsterdam, \\ The Netherlands
}

\begin{abstract}
To accurately determine sample optical properties using single fiber reflectance spectroscopy (SFR), an absolute calibration of the reflectance is required. We investigated two SFR calibration methods, using a calibrated mirror and using the Fresnel reflection at the fiber tip as a reference. We compared these to commonly used calibration methods, using either Intralipid-20\% in combination with Monte Carlo simulations or Spectralon as a reference. The Fresnel reflection method demonstrated the best reproducibility and yielded the most reliable result. We therefore recommend the Fresnel reflection method for the measured absolute reflectance calibration of SFR. $\odot$ The Authors. Published by SPIE under a Creative Commons Attribution 3.0 Unported License. Distribution or reproduction of this work in whole or in part requires full attribution of the original publication, including its DOI. [DOI: 10.1117/1.JBO.22.10.100502]
\end{abstract}

Keywords: single-fiber reflectance spectroscopy; calibration; measured absolute reflectance.

Paper 170455LRR received Jul. 11, 2017; accepted for publication Oct. 9, 2017; published online Oct. 30, 2017.

\section{Introduction}

Broadband fiberoptic spectroscopy is investigated for diagnostic applications, e.g., to noninvasively determine tissue scattering and absorption properties. Spectroscopic instrumentation requires a calibration to account for wavelength-dependent factors, such as the output from the fiber and detector sensitivity. For some diffuse reflectance spectroscopy ${ }^{1}$ techniques, a relative calibration of the reflectance is sufficient. For single fiber reflectance spectroscopy (SFR), however, the measured absolute reflectance $R$ is related to the sample optical properties ${ }^{2,3}$

$R=\eta_{\text {limit }} \cdot f\left(\mu_{s}^{\prime}, \mu_{a}, \gamma, d_{\mathrm{fib}}\right)$,

$\eta_{\text {limit }}=\left(\frac{\mathrm{NA}}{n_{\text {sample }}}\right)^{2}$,

where $f\left(\mu_{s}^{\prime}, \mu_{a}, \gamma, d_{\mathrm{fib}}\right)$ describes the reflectance of the sample as a function of the reduced scattering coefficient $\mu_{s}^{\prime}$, the absorption coefficient $\mu_{a}$, a parameter related to the scattering phase function $\gamma$, and the fiber diameter $d_{\text {fib }} . R$ also depends on the lower limit of the collection efficiency of the fiber, $\eta_{\text {limit }}$, which

*Address all correspondence to: Xu U. Zhang, E-mail: xu.zhang@amc.uva.nl depends on the fiber numerical aperture (NA) and the refractive index of the sample $n_{\text {sample. }}$. For high values of $\mu_{s}^{\prime} d_{\text {fib }}, R$ reaches the upper limit $\eta_{\text {limit }} \cdot{ }^{2}$ Consequently, to extract tissue optical properties using SFR, an absolute calibration of $R$ is required. However, no standard method exists to quantitate $R$ of a certain sample using SFR.

One common calibration approach uses Intralipid-20\% as a reference. The expected reflectance spectrum ${ }^{4}$ of Intralipid-20\% is determined using Monte Carlo (MC) simulations, which requires wavelength-dependent optical properties as input. Therefore, the accuracy of the input optical properties directly determines the accuracy of this approach.

Another commonly applied calibration approach uses the diffuse reflectance standard Spectralon as a reference ${ }^{5}$ - a flat solid Lambertian reflector, which has roughly $99 \%$ reflectivity. It is assumed that $R$ of Spectralon equals $\eta_{\text {limit }}$ [Eq. (2)]. However, because the reduced scattering coefficient of Spectralon is unknown, the validity of this assumption is not known either.

Our aim is to develop a method to calibrate $R$ of SFR that only requires well established data on the reference sample and/or the measurement setup. In this paper, we will compare the two commonly applied calibration methods using (1) Spectralon, (2) diluted Intralipid-20\% combined with MC simulations with two methods using (3) a calibrated mirror and (4) the Fresnel reflection at the fiber-air interface.

\section{Methods}

White light was emitted by a halogen light source (Ocean Optics, HL-2000) and coupled through the first branch of a bifurcated fiber into a multimode measurement fiber and onto the sample. A fraction of the light was scattered back into the measurement fiber and guided back through the other branch of the bifurcated fiber into the spectrometer (Avantes, AvaspecULS2048L Starline) (Fig. 1).

Each calibration method was performed using a $400-\mu \mathrm{m}$ silica measurement fiber ${ }^{6}$ (NA $0.22 \pm 0.02$ ). The measurement fiber was polished flat with subminiature version A connectors on both ends. To facilitate comparison between the calibration methods, we used undiluted Intralipid- $20 \%$ as a measurement sample for all methods. $R$ of undiluted Intralipid-20\% was calculated as

$R_{\text {sample }}=R_{\text {ref }} \times \frac{\left(I_{\text {sample }}-I_{\text {sampleback }}\right)}{\left(I_{\text {ref }}-I_{\text {ref back }}\right)}$.

The background intensities $I_{\text {sampleback }}$ and $I_{\text {refback }}$ were subtracted from the measured signal intensities of the sample $I_{\text {sample }}$ and reference $I_{\text {ref }}$, respectively, to account for the dark current of the spectrometer and the internal reflection at the fiber-medium interface due to the refractive index mismatch. One set of measurements including reference, sample, and background measurements yielded one measured absolute reflectance value. The background was measured once per set due to the small intensity variation of the system noise. To test the reproducibility, we cleaned the fiber tip, repositioned it in the sample and reference, and disassembled and reassembled the connector. To reduce ambient light, measurements were performed in a dark room.

\subsection{Spectralon}

The fiber surface was in contact with Spectralon, with a drop of demineralized water in between to improve optical contact and 


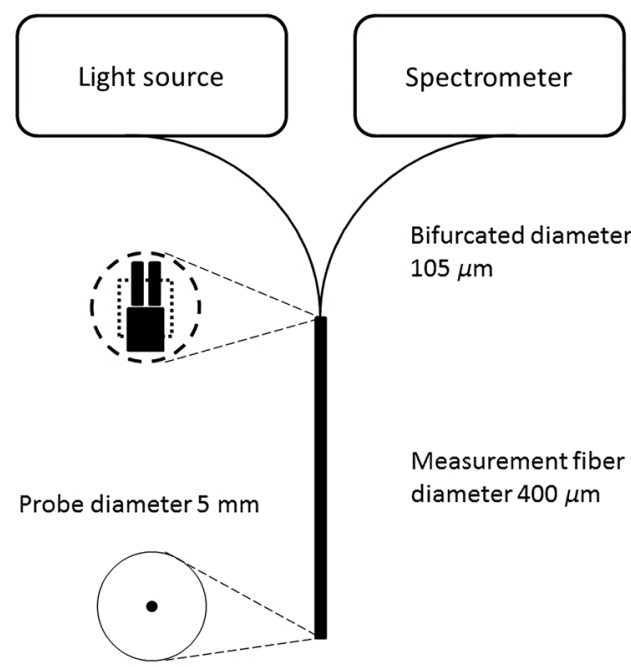

Fig. 1 Schematic of SFR.

reduce internal reflection. The measured absolute reflectance of the undiluted Intralipid-20\% sample was calculated using Eq. (3), assuming $R_{\text {ref }}$ equals $\eta_{\text {limit }}$. $\eta_{\text {limit }}$ was calculated using Eq. (2) with an NA that equals 0.22 and $n_{\text {medium }}$ that equals the refractive index of water. Both $I_{\text {sampleback }}$ and $I_{\text {refback }}$ were the measured intensity of water in a black container.

\subsection{Monte Carlo Simulations and Diluted Intralipid- $20 \%$}

We used the MC code previously described by Post et al., ${ }^{7}$ to simulate $R$ of Intralipid-20\%. Since the phase function of Intralipid-20\% is only available for diluted samples, we simulated and measured on 1:20 diluted Intralipid-20\% to avoid concentration-dependent effects on the scattering cross section and phase function. ${ }^{8}$ Simulations were performed for the wavelength range in increments of $20 \mathrm{~nm}$. The fiber was submerged in diluted Intralipid-20\%. We used the refractive index of water for the sample and the layer above the sample. The fiber NA was set to 0.22 for all wavelengths. Photons were launched from uniformly distributed locations at the fiber tip with launch angles uniformly distributed within the acceptance angle of the fiber. Photons hitting the fiber with an angle within the acceptance angle of the fiber were counted. The acceptance angle was calculated as the arcsine of the ratio of the fiber NA and $n_{\text {water }}$. We used the wavelength-dependent reduced scattering coefficient and phase function from Michels et al. ${ }^{9}$ Recently, the same group optimized their measurement setup and obtained different results for the phase function, which were only published for $635 \mathrm{~nm} .{ }^{10}$ To investigate the difference between using the previously published wavelength-dependent phase function and the updated phase function at $635 \mathrm{~nm},{ }^{10}$ we performed three sets of simulations with each phase function. The spectra of the absorption coefficient ${ }^{11}$ were calculated based on the composition of our diluted sample.

We measured both diluted $I_{\text {ref }}$ and undiluted Intralipid-20\% $I_{\text {sample. }} R$ of undiluted Intralipid-20\% was calculated using Eq. (3), where $R_{\text {ref }}$ was the measured absolute reflectance of Intralipid-20\% dilution obtained using MC simulations. Both $I_{\text {sampleback }}$ and $I_{\text {refback }}$ were the measured intensity of water in a black container.

\subsection{Calibrated Mirror}

A calibrated mirror (calibrated to an NIST master standard) was used as a reference (Ocean Optics; STAN-SSH-NIST). The fiber surface was aligned in contact with the mirror, with a drop of demineralized water in between to relate reflected intensity directly to the calibrated reflectivity of the mirror. We prefer this arrangement over a noncontact setup for experimental simplicity, even though the alignment remains challenging. $R$ of the undiluted Intralipid-20\% sample was calculated using Eq. (3), where $R_{\text {ref }}$ was the calibrated reflectivity of the mirror supplied by the manufacturer and both $I_{\text {sampleback }}$ and $I_{\text {ref back }}$ were the measured intensity of water in a black container.

\subsection{Fresnel Reflection}

The Fresnel reflection at the fiber-air interface was used as a reference. The range of incident angles at the fiber tip in our setup was small enough (roughly within $10 \mathrm{deg}$, based on NA, refractive index of air, and Snell's law) so that the Fresnel reflection is insensitive to the incident angle, simplifying the Fresnel equations to

$R_{\text {sample }}=\frac{\left(n_{\text {eff }}-n_{\text {sample }}\right)^{2}}{\left(n_{\text {eff }}+n_{\text {sample }}\right)^{2}}$.

However, the effective index of the fiber, $n_{\mathrm{eff}}$, was not supplied by the manufacturer. To obtain $n_{\text {eff }}$, we measured the reflected intensities of air $I_{\text {air }}$ and water $I_{\text {water }}$, respectively. The ratio between the Fresnel reflection for air $R_{\text {air }}$ and water $R_{\text {water }}$ can be expressed as follows:

$\frac{R_{\text {air }}}{R_{\text {water }}}=\frac{I_{\text {air }}-I_{\text {dark }}}{I_{\text {water }}-I_{\text {dark }}}$,

where $I_{\text {dark }}$ accounts for the dark current of the spectrometer, which was measured by blocking the light source. Combining Eqs. (4) and (5), $n_{\text {eff }}$ was calculated. The measured absolute reflectance of the undiluted Intralipid-20\% was calculated using Eq. (3), where $R_{\text {ref }}$ was the Fresnel reflection at the fiber-air interface $R_{\text {air }}$, which was calculated using Eq. (4) and the calculated $n_{\text {eff }}$. $I_{\text {sampleback }}$ was the measured intensity of water in a black container and $I_{\text {refback }}$ was $I_{\text {dark }}$.

\subsection{Data Analysis}

Five sets of measurements were performed with each method. Results are shown as mean \pm one standard deviation (SD). For MC simulations combined with Intralipid-20\%, the variation was calculated as the sum of the relative errors of the MC simulations and the measurements. We analyzed our data from 400 to $900 \mathrm{~nm}$.

\section{Results}

Using the Fresnel reflection method [Eqs. (4) and (5)], the effective index of the fiber was calculated, which differed from the refractive index of fused silica ${ }^{12}$ with $1 \%$ to $2 \%$ (Fig. 2).

The measured absolute reflectance spectra of undiluted Intralipid-20\% obtained with all four methods are shown in Fig. 3.

$R$ values based on the Fresnel reflection, the calibrated mirror and the MC simulations with both the phase functions were similar. Spectralon resulted in a much higher reflectance and MC 


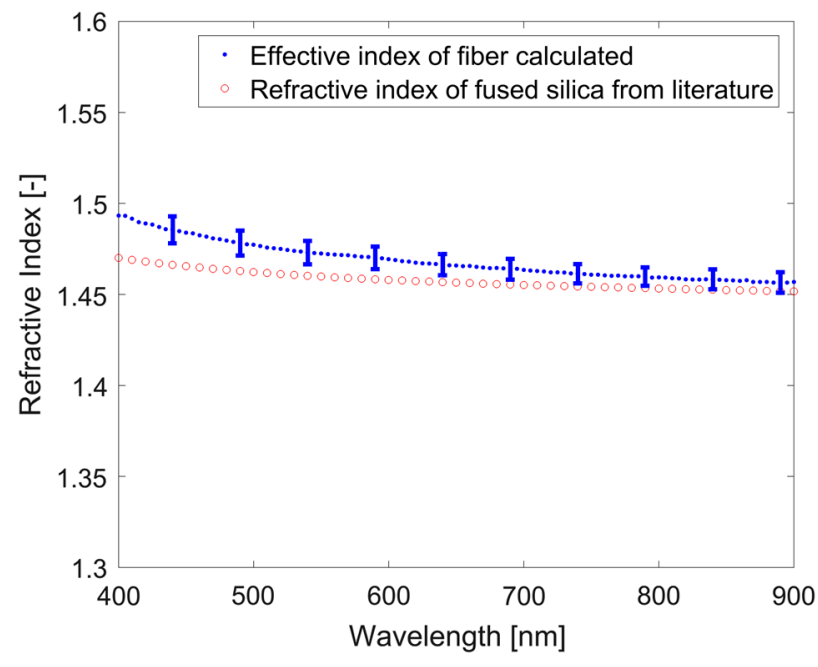

Fig. 2 Refractive index of fused silica from literature and the calculated effective index of the fiber.

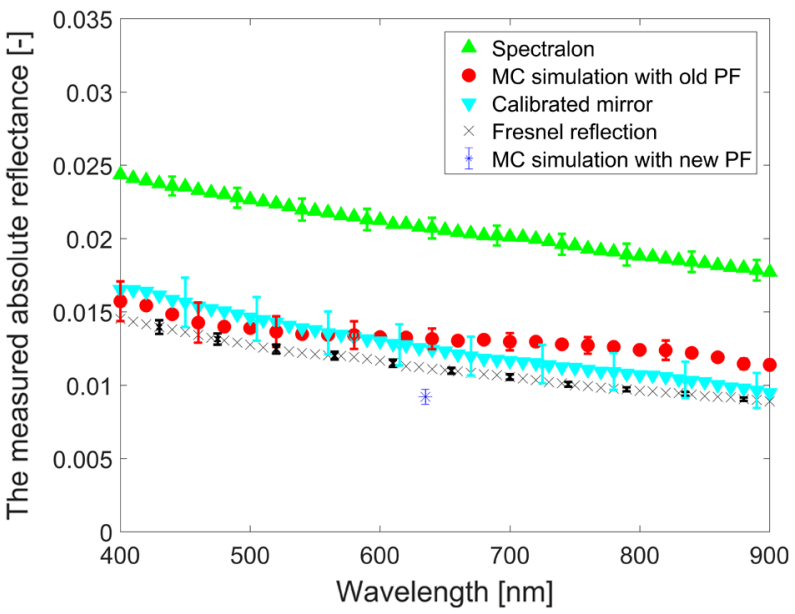

Fig. 3 The measured absolute reflectance of undiluted Intralipid-20\% obtained using different methods.

Table $1 R$ of undiluted Intralipid- $20 \%$ at $635 \mathrm{~nm}$ obtained using different calibration methods.

\begin{tabular}{lccc}
\hline & Mean & SD & SD/mean (\%) \\
\hline Spectralon & 0.0208 & 0.0007 & 3.4 \\
MC + old PF & 0.0132 & 0.0007 & 5.3 \\
Mirror & 0.0125 & 0.0014 & 11.2 \\
Fresnel & 0.0112 & 0.0002 & 1.8 \\
MC + new PF & 0.0092 & 0.0005 & 5.4 \\
\hline
\end{tabular}

simulations with the updated phase function resulted in the lowest reflectance at $635 \mathrm{~nm}$. Comparing the results at $635 \mathrm{~nm}$ (Table 1), the Fresnel reflection method demonstrated to have the lowest SD.

\section{Discussion}

We compared the obtained $R$ of undiluted Intralipid-20\% using four different calibration methods (Spectralon, diluted
Intralipid-20\% combined with MC simulations, a calibrated mirror, or the Fresnel reflection at the fiber-air interface as a reference). There is a large difference between the results obtained using Spectralon and the other methods, which indicates that the assumption that the reflectance of Spectralon equals $\eta_{\text {limit }}$ may be wrong. The difference between the results obtained using the other methods is smaller. The MC simulations with the updated phase function resulted in the lowest reflectance value compared to the other methods, which can be the result of errors in the input optical properties of the Intralipid-20\% dilution, which is depicted by the factor of 1.4 discrepancies between the results using the old and updated phase function. The calibration methods using the calibrated mirror or the Fresnel reflection do not require assumptions on the optical properties of the reference sample and only need well-established data as input. The difference between the results obtained with the calibrated mirror and the Fresnel reflection method (12\%) can be explained by the loss of reflected light from the mirror surface due to the difficulty in aligning the fiber and mirror surfaces. This misalignment will lead to a decrease in $I_{\text {ref }}$ and results in an overestimation of $R$ of undiluted Intralipid- $20 \%$.

The variation of the reflectance measurement results was different for the different calibration methods. The Fresnel reflection method had the best reproducibility. With the calibrated mirror as a reference, the variable contact between the fiber and the mirror was an issue, which resulted in the worst reproducibility. Furthermore, the measurements can damage both the mirror and the fiber surface, resulting in less accurate measurements. For the Spectralon-based method, the variability can be explained by the variable contact quality between the fiber and the Spectralon. For the MC simulation method, the variation in the simulated reflectance was low $(<3 \%)$. However, the reference measurements using diluted Intralipid-20\% had a relatively large variation, which we attribute to the relatively low signal.

Overall, comparing these four calibration methods, we recommend using the Fresnel reflection method for the measured absolute reflectance calibration of SFR. First, the Spectralon method requires the knowledge of both the NA of the fiber and the reduced scattering coefficient of Spectralon. Although the calibration with Intralipid-20\% combined with MC simulations does not require the knowledge of NA to obtain the reflectance of the sample [ $f$ in Eq. (1)], it does require the knowledge of NA to obtain the measured absolute reflectance [ $R$ in Eq. (1)]. In addition, the accuracy significantly depends on the accuracy of the input optical properties. The calibrated mirror and Fresnel reflection method only require well-established data (the NA of the fiber, reflectivity of the mirror, and refractive indices of air and water). Second, compared to the mirror calibration, the reproducibility of the Fresnel method is higher. The discrepancy between the refractive index of fused silica and the obtained effective index of the fiber (Fig. 2) can be caused by the fact that the former was measured for bulk material but not for fibers. The accuracy was checked by using it as an input to determine the refractive index of ethanol based on the Fresnel reflection at fiber tip. The result differed $<0.2 \%$ from literature.

To use the Fresnel reflection as a calibration method, the fiber tip has to be polished flat while SFR measurements are usually performed with the fiber polished at an angle to minimize the internal reflection. Preliminary results on the comparison between the flat-polished probe and probe polished at $15 \mathrm{deg}$ did not show significant difference on collection manner. To 


\section{JBO Letters}

implement the Fresnel reflection method in daily SFR measurements, one could use a flat polished probe with the Fresnel reflection method to quantitate $R$ of undiluted Intralipid-20\% and use the latter as a routine reference sample with an angle-polished probe made from the fiber of the same batch to quantitate $R$ of other samples. Intralipid-20\% is a strictly controlled liquid medical product and the optical properties variation is small between batches. ${ }^{13}$ Furthermore, this quick and simple approach can be done without compromising the sterility of the fiber, which is essential in many clinical studies.

\section{Conclusion}

We compared four methods to perform an absolute calibration for SFR measurements. The Fresnel reflection method yields reproducible and reliable measured absolute reflectance values for SFR, and the accuracy does not depend on the assumed or input optical properties of a reference sample. Furthermore, this method only requires the refractive indices of water and air and the NA of the fiber as input, which are well established. Therefore, we recommend to use this method to calibrate the measured absolute reflectance for SFR measurements.

\section{Disclosures}

No conflicts of interest, financial or otherwise, are declared by the authors.

\section{Acknowledgments}

This research was supported by the Netherlands Organization for Scientific Research (Technology Foundation NWO-TTW, iMIT-FIBER Grant No. 12702).

\section{References}

1. N. Bosschaart et al., "Optical properties of neonatal skin measured in vivo as a function of age and skin pigmentation," J. Biomed. Opt. 16(9), 097003 (2011).
2. S. C. Kanick et al., "Method to quantitatively estimate wavelength-dependent scattering properties from multidiameter single fiber reflectance spectra measured in a turbid medium," Opt. Lett. 36(15), 2997-2999 (2011).

3. P. R. Bargo, S. A Prahl, and S. L. Jacques, "Collection efficiency of a single optical fiber in turbid media for reflectance spectroscopy," in OSA Biomedical Topical Meetings, pp. 604-606 (2002).

4. U. A. Gamm et al., "Quantification of the reduced scattering coefficient and phase-function-dependent parameter $\gamma$ of turbid media using multidiameter single fiber reflectance spectroscopy: experimental validation," Opt. Lett. 37(11), 1838 (2012).

5. S. C. Kanick, H. J. C. M. Sterenborg, and A. Amelink, "Empirical model of the photon path length for a single fiber reflectance spectroscopy device," Opt. Express 17(2), 860-871 (2009).

6. Fiber specification, http://www.literature.molex.com/SQLImages/ kelmscott/Molex/PDF_Images/987650-8934.pdf (2013).

7. A. L. Post et al., "Modeling subdiffusive light scattering by incorporating the tissue phase function and detector numerical aperture," J. Biomed. Opt. 22(5), 050501 (2017).

8. P. Di Ninni, F. Martelli, and G. Zaccanti, "Effect of dependent scattering on the optical properties of Intralipid tissue phantoms," Biomed. Opt. Express 2(8), 2265-2278 (2011).

9. R. Michels, F. Foschum, and A. Kienle, "Optical properties of fat emulsions," Opt. Express 16(8), 5907 (2008).

10. F. Foschum and A. Kienle, "Optimized goniometer for determination of the scattering phase function of suspended particles: simulations and measurements," J. Biomed. Opt. 18(8), 085002 (2013).

11. R. Nachabé et al., "Validation of interventional fiber optic spectroscopy with MR spectroscopy, MAS-NMR spectroscopy, high-performance thin-layer chromatography, and histopathology for accurate hepatic fat quantification," Invest. Radiol. 47(4), 209-216 (2012).

12. I. H. Malitson, "Interspecimen comparison of the refractive index of fused silica," J. Opt. Soc. Am. 55(10), 1205-1209 (1965).

13. P. Di Ninni, F. Martelli, and G. Zaccanti, "Intralipid: towards a diffusive reference standard for optical tissue phantoms," Phys. Med. Biol. 56(2), N21-N28 (2011). 